Service social

\title{
Oppression, intolérance et intervention : facettes d'une pratique à paramètres multiples
}

\section{Gérald Doré}

Volume 40, numéro 3, 1991

Oppression, intolérance et intervention

URI : https://id.erudit.org/iderudit/706540ar

DOI : https://doi.org/10.7202/706540ar

Aller au sommaire du numéro

Éditeur(s)

École de service social de l'Université Laval

ISSN

1708-1734 (numérique)

Découvrir la revue

Citer ce document

Doré, G. (1991). Oppression, intolérance et intervention : facettes d'une

pratique à paramètres multiples. Service social, 40(3), 3-8.

https://doi.org/10.7202/706540ar d'utilisation que vous pouvez consulter en ligne.

https://apropos.erudit.org/fr/usagers/politique-dutilisation/ 


\section{AVANT-PROPOS}

\section{Oppression, intolérance et intervention : facettes d'une pratique à paramètres multiples}

Gérald Doré

Dans I'article qui ouvre ce numéro thématique, Lise Noël définit bien les deux premiers termes du titre : oppression et intolérance. L'oppression est la " pratique, c'est-à-dire, ajouterions-nous, la réalité vécue des rapports concrets d'exploitation économique, de domination politique et d'aliénation culturelle tels qu'ils s'inscrivent dans la vie quotidienne de nos sociétés. L'intolérance est la " théorie ", c'està-dire, précise-t-elle elle-même, "le discours attestataire, le fondement théorique qui prétend justifier l'oppression ou les relations de domination".

Son livre L'intolérance. Une problématique générale, prix du Gouverneur général 1989, fait ici l'objet d'une recension. II marque, au Québec, un temps fort dans une réflexion qui se mène à l'échelle internationale sur l'articulation des différentes formes d'oppression, sur les paramètres d'identité autour desquels elles se construisent et sur les discours qui les légitiment. Outre les nombreuses références par paramètre d'identité (âge, classe sociale, état de santé, ethnicité et race, orientation sexuelle et sexe), sa bibliographie nous propose une série d'ouvrages traitant de l'oppression à " paramètres multiples " (Noël, 1989 : 304-306).

Dans la " présentation théorique " par laquelle il suggère de terminer le jeu de simulation auquel nous initie son article, Maurice Moreau met en valeur un apport non mentionné par Noël sur le même sujet : celui de Wineman (1984). L'ouvrage de Albert, Cagan, Chomsky, Hahnel, King, Sargent et Sklar, Liberating Theory (1986), est une autre contribution pertinente. Du côté français, on peut citer le 
livre de Balibar et Wallerstein, Race, nation, classe. Les identités ambiguës (1988).

L'intervention est le troisième terme du titre qui nous interpelle. En guise $d^{\prime}$ introduction à son article, Maurice Moreau retrace les ouvrages qui depuis 1970, dans le milieu anglo-saxon du travail social, ont insisté pour que les travailleuses sociales et les travailleurs sociaux " reconnaissent les liens entre les statuts sociaux des clientes et clients infériorisés, les conditions idéologiques et matérielles auxquelles ils sont soumis et les impacts sur leur comportement... ». Pour le seul Québec francophone, si nous procédions ici par catégorie spécifique d'oppression, nous aurions à mettre en évidence nombre de publications relatives à l'intervention auprès des enfants, des jeunes et des personnes âgées, à l'animation de groupes populaires, au travail avec des handicapés physiques ou intellectuels, hommes et femmes, avec des communautés culturelles, avec des homosexuels et des lesbiennes et, enfin, à l'intervention féministe, qui s'est taillée une place importante et reconnue au cours des deux dernières décennies.

Par ailleurs, pour ce qui est de la prise en compte de l'articulation d'oppressions multiples dans l'intervention, il s'agit d'un ordre de préoccupation déjà présent dans la pratique, mais peu explicité sur le plan théorique. Dans l'approche de conscientisation, clairement identifiée à une problématique des rapports d'oppression, dans laquelle la classe sociale occupe le statut d'universalité attribué au sexe par Lise Noël dans son article, une place est faite à la question de l'articulation entre oppression " sociale » (liée à la classe sociale) et oppression dite spécifique (toute forme d'oppression traversant les strates de la " pyramide sociale »). La conscientisation reconnaît "l'existence d'oppressions spécifiques (fondées sur le sexe, l'orientation sexuelle, l'ethnie, la couleur de la peau, etc.) qui traversent les classes et se surajoutent pour diviser et rediviser les groupes dominés " (Ampleman et al., 1983 : 269). Dans l'approche structurelle, à laquelle se rattachait Maurice Moreau, la perspective est plutôt holistique. Comme il le signale dans son article, celle-ci « suggère que les différentes formes d'oppression se croisent et se chevauchent... " Dans l'optique de Wineman, cette perspective devrait déboucher sur " un vaste mouvement radical composé de coalitions inter-reliées dans lequel les individus et les groupes peuvent concentrer leurs efforts sur la lutte contre les formes d'oppression qui les touchent le plus profondément... » (Wineman, $1984:$ 198).

Dans ce numéro thématique sur "Oppression, intolérance et intervention ", le concept de cette coalition représente l'horizon à ne pas perdre de vue, plutôt que le cadre de référence immédiat. Notre propos est en effet plus modeste, mais correspond sans doute au 
moment actuel, dans l'élaboration d'une stratégie d'intervention fondée sur une problématique de l'oppression et de l'intolérance.

Outre la conceptualisation générale à laquelle nous nous sommes déjà référés, l'article de l'historienne Lise Noël soulève la question des causes des phénomènes d'intolérance et d'oppression et cherche à déterminer s'il existe une oppression plus fondamentale que les autres. Elle nous explique pourquoi, selon elle, les rapports de domination fondés sur le sexe sont, non pas plus fondamentaux, mais plus universels que les autres.

Travailleuse sociale et organisatrice communautaire de formation, agente de relations humaines dans un centre de services sociaux, Louise Lebœuf traite de la double oppression liée au sexe et à la classe sociale qui caractérise la clientèle majoritaire des services sociaux : femmes et pauvres. À partir d'une recherche du Conseil national du bien-être social, elle met notamment en valeur comment l'ajout d'un troisième paramètre d'oppression est associé à une pauvreté accrue : femme autochtone, femme handicapée, femme immigrante, femme appartenant à une minorité visible. Elle rend compte d'une démarche d'intervention qu'elle a réalisée avec des groupes sur le thème de la pauvreté des femmes. Elle se situe enfin comme femme et comme intervenante dans la lutte pour la libération des femmes.

Psychologue et lui-même originaire du Village-des-Hurons, Wendake, Raymond Sioui nous trace une rétrospective du processus historique qui a conduit à la domination des Premières nations au Canada. II insiste sur la longue période d'infériorisation qui a précédé les années récentes et dégage les symptômes associés à l'oppression amérindienne. Il est fort probable, selon lui, que ces symptômes présentent de grandes affinités avec ceux qu'éprouvent les groupes soumis à d'autres formes d'oppression. L'auteur passe ensuite en revue diverses formes d'action collective entreprises par les communautés amérindiennes pour se libérer de l'oppression qu'elles subissent, notamment la prise en charge de leurs services sociaux et la revalorisation de leur culture. Il convie en conclusion son lecteur à réaliser l'effort d'accommodation, au sens où l'entend Piaget, par lequel nous pourrions nous affranchir des rivalités.

L'article de Robert Arsenault présente une actualisation de l'approche de conscientisation précédemment évoquée. Travailleur social de formation et agent de réinsertion sociale dans le service de santé mentale d'un centre hospitalier en région, l'auteur témoigne de sa pratique avec un groupe de parents de personnes psychiatrisées et avec un groupe de personnes elles-mêmes atteintes de maladie mentale. Il fait ressortir la dynamique d'oppression vécue par ces personnes dans un contexte où jouent des facteurs structurels comme le pouvoir institutionnel sur la maladie et les contraintes découlant du 
recours à l'aide sociale. Il décrit la démarche qu'il a mise en œuvre avec deux autres intervenants, dans le but de donner aux personnes concernées l'occasion de devenir des sujets agissant sur les éléments qui les oppriment.

Avec l'article d'André Jacob, professeur au Département de travail social de l'Université du Québec à Montréal, le thème de l'oppression liée à l'ethnicité est de nouveau abordé, mais en rapport avec une situation existentielle très différente de celle des Premières nations. L'auteur traite la question des personnes réfugiées, en confrontant le discours et la réalité. Ici, le discours officiel n'est pas ouvertement attestataire d'intolérance. Il est ambivalent et même plutôt humanitaire. La réalité, elle, est oppressive, notamment à travers le caractère sélectif des procédures, leur longueur et leur lourdeur bureaucratique, leur tendance discriminatoire une fois les personnes entrées au pays. En ce qui concerne les services sociaux spécialisés à leur disposition, l'auteur constate qu'ils relèvent pour la plupart d'organismes non gouvernementaux, obligés de composer avec un sous-financement chronique. Pour continuer à être financés, ils doivent " prouver leur efficacité en offrant des services dans des conditions qui seraient jugées inacceptables par des professionnels et des employés du gouvernement ". Ils doivent aussi pour les mêmes raisons " se plier à une forme de soumission idéologique et politique ». L'auteur identifie ici le lieu d'un partenariat à redéfinir.

L'article que je signe pour ma part concerne les liens que j'ai gardés avec la pratique de l'organisation communautaire, dans le cours de mon emploi de professeur à l'École de service social de l'Université Laval. C'est un témoignage d'intervenant sur l'enjeu complexe de la potentialisation politique des milieux où se recrute en majorité la clientèle des services sociaux et où les organisateurs et organisatrices communautaires déploient le plus souvent leur pratique d'animation des collectivités. Je passe en revue les diverses expériences, tant sur le plan municipal que sur le plan national québécois, dans lesquelles s'est manifestée une connivence entre organisation communautaire et politique partisane, dans le sens de cette potentialisation. Je propose une problématique théorique de l'exclusion politique des classes populaires, du point de vue de la culture des gens qui la vivent, et en tenant compte des résultats d'une recherche en développement. Je conclus en abordant sous ses différentes facettes la question de l'engagement politique des organisateurs et organisatrices communautaires, dans les circonstances actuelles de la pratique.

Jusqu'à son décès survenu le 13 novembre 1990, Maurice Moreau était professeur à l'École de service social de l'Université de Montréal. Cité jusqu'ici pour ses apports théoriques, son article est une contribution pédagogique majeure pour l'enseignement de l'empathie à 
l'égard des minorités opprimées, dans la formation en travail social. Son caractère novateur tient au traitement simultané de paramètres multiples : race, classe sociale, sexe, orientation sexuelle, santé, âge, origine ethnique, nationalité, état matrimonial et religion. La mise en situation qu'il induit permet d'atteindre le niveau des attitudes, si crucial en ce qui concerne la gestation et le développement des intolérances. Son article est ici publié pour sa valeur intrinsèque, mais en même temps en hommage à la mémoire d'un collègue estimé pour sa compétence et son engagement dans les enjeux qui font le thème de ce numéro.

Les deux derniers textes, I'article de Donald Baillargeon, politicologue et chercheur autonome, et la note de recherche de JeanLouis Gendron et Myra Piat, respectivement professeur et étudiante au doctorat à l'École de service social de l'Université Laval, se rapportent à un problème particulier auquel ont dû faire face plusieurs intervenantes et intervenants œuvrant dans le développement de ressources: l'intolérance des communautés locales à l'égard de l'implantation de ressources pour des clientèles identifiées à des paramètres d'oppression. À partir des résultats d'une recherche empirique menée avec d'autres chercheurs, Donald Baillargeon pose le problème de la légitimation de la cohabitation sociale et des alliances à développer en sa faveur. II préconise une révision en profondeur de la stratégie $d^{\prime} i m$ plantation des projets et de gestion des conflits pour qu'elle corresponde mieux aux différents défis communautaires à relever. Pour leur part, Jean-Louis Gendron et Myra Piat font le point sur l'état de la recherche américaine sur le même sujet. Ils constatent que les analyses d'attitudes tendent à dominer la recherche, qu'une résistance collective peut être prédite à partir de certaines variables et que l'intolérance est favorisée par une trop grande concentration de ressources. Des données sont aussi disponibles concernant le type de clientèle, le type de voisinage et le type de ressources, en rapport avec le degré $d^{\prime}$ intolérance. Ils notent également l'absence d'études systématiques sur le processus ou la montée de l'intolérance, de même que la pauvreté de la recherche québécoise en la matière, mis à part la recherche de Baillargeon et al. déjà citée et quelques autres travaux. Une riche bibliographie accompagne la note de recherche.

Quand le projet de ce numéro thématique a été lancé, nous ne nous attendions pas à l'unité et à la complémentarité de pensée et d'action que révèle le travail fini. Dans un climat social réputé en proie à la démobilisation et au désenchantement à l'égard des grands projets et des grandes causes, il est stimulant de constater que des forces de résistance à l'oppression et à l'intolérance continuent d'être à l'œuvre, dans tous les champs d'activité où elles se manifestent. Des modèles d'intervention sont en gestation, en expérimentation ou en action, qui 
prennent en considération les stigmates de marginalisation dont sont victimes les collectivités d'où viennent la majorité des personnes qui s'adressent aux services sociaux. On est loin du " vaste mouvement " espéré par Wineman, mais il est encourageant de prendre conscience qu'en des milieux aussi variés que ceux évoqués dans les articles de ce numéro des hommes et des femmes sont " en mouvement " vers cette visée que la conscientisation cherche à résumer dans l'énoncé suivant : "libération personnelle et collective des exploitations économiques, des dominations politiques et organisationnelles, des aliénations culturelles et religieuses et de l'idéologie dominante" (Ampleman et al., $1983: 269$ ). À cet effet, ce numéro ne contient pas que des connaissances, mais aussi des motivations pour l'action.

\section{Références bibliographiques}

Albert, M., L. Cagan, N. Chomsky, R. Hahnel, M. King, L. Sargent et H. Sklar (1986). Liberating Theory. Boston : South End Press.

ampleman, G., G. Doré, L. Gaudreau, C. Larose, l. Lebceuf et D. Ventelou (1983). Pratiques de conscientisation. Montréal : Nouvelle Optique.

BAlibar, E. et I. WALlerstein (1988). Race, nation, classe. Les identités ambiguës. Paris : La Découverte.

NoËL, L. (1989). L'intolérance. Une problématique générale. Montréal : Boréal. Wineman, S. (1984). The Politics of Human Services : Radical Alternative to the Welfare State. Montréal : Black Rose Books. 